\title{
Valérie Raby
}

(1967-2019)

Jean-Marie Fournier

\section{OpenEdition}

\section{Journals}

Édition électronique

URL : https://journals.openedition.org/hel/458

DOI : $10.4000 /$ hel. 458

ISSN : 1638-1580

Éditeur

Société d'histoire et d'épistémologie des sciences du langage

Édition imprimée

Date de publication : 28 septembre 2020

Pagination : 3-5

ISSN : 0750-8069

Référence électronique

Jean-Marie Fournier, « Valérie Raby », Histoire Épistémologie Langage [En ligne], 42-1 | 2020, mis en ligne le 28 octobre 2021, consulté le 29 octobre 2021. URL : http://journals.openedition.org/hel/458 DOI : https://doi.org/10.4000/hel.458

Ce document a été généré automatiquement le 29 octobre 2021

\section{(c) $($ i) $(9)$}

HEL is licensed under a Creative Commons Attribution-NonCommercial-NoDerivatives 4.0 International License 


\section{Valérie Raby}

(1967-2019)

\section{Jean-Marie Fournier}

\section{Valérie Raby}

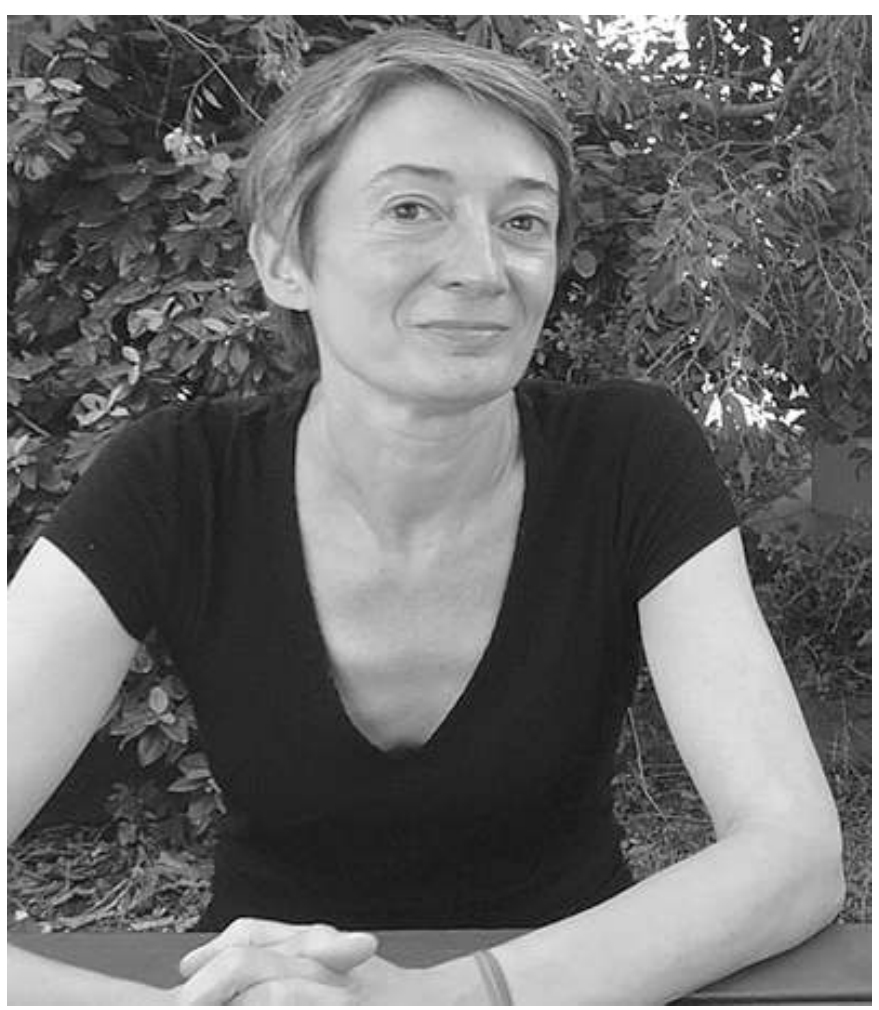

La disparition brutale de Valérie nous laisse en état de choc et de sidération.

2 Le vide qu'elle laisse est immense et les mots sont insuffisants pour dire qui elle était, ce qu'elle représentait pour nous et ce qu'elle a accompli.

3 Il y a surtout une cruauté insoutenable à devoir aujourd'hui retracer son parcours académique interrompu parce que l'ordre des choses aurait dû être qu'il se prolonge 
longtemps et qu'elle accomplisse les projets qu'elle dessinait dans sa vie de chercheuse et de professeure récemment élue à la Sorbonne Nouvelle.

Il y a une ironie tragique à devoir prononcer les mots convenus du deuil, pour elle qui l'était si peu, qui déjouait toujours d'un trait d'ironie souriante, jamais blessant, ou d'un éclat de rire aux notes graves, ce qui précisément est convenu ou empesé dans les discours et les postures.

5 Valérie a été secrétaire de la SHESL de 2008 à 2014, elle en était la vice-présidente depuis 2014.

6 Elle a commencé à codiriger HEL avec Jean-Luc Chevillard en juin 2012, puis elle en a assuré seule la direction à partir de janvier 2015. Elle s'est acquittée de cette direction impeccablement, insufflant avec une fermeté et une efficacité tranquilles vigueur et stabilité à notre revue.

7 Son parcours était celui, classique, de plusieurs d'entre nous: elle avait obtenu l'agrégation de lettres modernes de 1990 et occupé différents postes dans l'enseignement secondaire en collège puis en lycée pendant une dizaine d'années.

Parallèlement, elle a suivi la formation en sciences du langage de l'université Paris 7 où elle a d'abord obtenu une maîtrise (1995), puis un DEA de linguistique théorique et formelle avant de s'inscrire en doctorat sous la direction de Sylvain Auroux en 1997 et soutenir sa thèse trois ans plus tard en 2000 (une thèse en histoire des théories linguistiques bouclée en trois ans c'est rare !). C'est à partir de cette date qu'elle a donc été accueillie dans le laboratoire HTL et que nous l'avons croisée régulièrement au séminaire de Sylvain Auroux au septième étage de la tour centrale de Jussieu.

9 Elle a été recrutée l'année suivante en 2001 comme maître de conférences à l'université de Champagne-Ardennes où elle a occupé ce poste jusqu'en 2008 ; date à laquelle elle a obtenu sa mutation pour l'université Paris Sorbonne.

10 Enfin elle a soutenu son HDR en 2017 et a été recrutée comme professeure de linguistique française à la Sorbonne Nouvelle en 2018.

11 Pendant une vingtaine d'années, elle a donc été partie prenante, comme participante ou comme coordinatrice non seulement de tous les projets du laboratoire inscrits dans le champ de la grammatisation du français et des vernaculaires européens, mais également de plusieurs programmes transversaux comme le programme « Grammaires étendues» de l'axe 7 du Labex sous la direction d'Émilie Aussant; ou le programme «Énoncé » qu'elle a coordonné ; ou encore l'axe "Grammaires et outils linguistiques » de l'organigramme du laboratoire qu'elle a codirigé avec Bernard Colombat.

12 Elle a été en particulier une cheville ouvrière infatigable et rigoureuse de ces projets éditoriaux de longue haleine et de grande ampleur que sont la base de données des grammaires françaises chez Garnier, l'édition critique des grammaires de la tradition française (elle a conduit à bien l'édition d'un des premiers ouvrages paru dans la collection mise en place chez Garnier), ou encore le Dictionnaire historique de la terminologie linguistique (DHTL), pour n'en citer que les principaux.

Elle a dirigé de nombreux numéros de revues et ouvrages collectifs.

14 Elle a publié un grand nombre d'articles sur des sujets très variés, dans lesquels on peut distinguer deux ensembles principaux :

i. d'un côté, des questions d'histoire des théories grammaticales, relatives notamment à la syntaxe dans les grammaires françaises (en particulier autour des notions de phrase et 
d'énoncé auxquelles elle a consacré sa thèse, plusieurs articles, un numéro de la revue Langages qu'elle a dirigé et une monographie de synthèse dont Jacques François a préparé le compte rendu dans le BSL; ou encore l'impersonnel, les complétives, l'interjection etc.) ;

ii. de l'autre, des questions d'épistémologie de la grammaire et de la linguistique: la rétrospection et ses enjeux, l'usage du discours historiographique par les grammairiens; l'écriture de l'histoire, la place des langues « rares » dans l'exemplification au XVIII siècle ; la question de l'extension du modèle latin dans la description des vernaculaires.

L'articulation dynamique de ces deux moments de son travail caractérise bien la démarche qui était la sienne et le développement de ses intérêts de recherche: d'une part, l'exploration approfondie d'un corpus et d'une tradition, l'exigence d'une documentation très précise et d'une érudition sans faille ; de l'autre, ensuite, la prise en charge de questions théoriques plus générales, cette réflexion étant elle-même nourrie du contact avec les travaux des collègues du laboratoire, dans ce contexte particulier d'interdisciplinarité et de cohésion théorique forte qui lui est propre et qu'elle avait singulièrement à cœur de contribuer à faire fructifier, convaincue que là s'édifiait une pensée originale de l'histoire et de l'épistémologie des sciences du langage. Elle attachait ainsi une grande importance aux activités et aux projets collectifs du laboratoire et de la SHESL ; elle a participé de façon décisive à la plupart de ceux qui en ont rythmé et structuré la vie depuis vingt ans (la revue et l'administration de la SHESL elle-même, une bonne part des colloques de la SHESL, les éditions successives des écoles d'été, la préparation d'ICHOLS 14 bien sûr). Elle était de celles et ceux sur qui on peut compter, toujours.

17 Elle était aussi une amie précieuse, unique, centrale dans la vie de plusieurs d'entre nous. Je lui dois personnellement beaucoup. Nous avons écrit en collaboration de nombreux articles. Rien de plus aisé, de plus plaisant, de plus simple que de travailler avec Valérie. Nous avions l'habitude presque chaque jour de réfléchir et d'aborder ensemble tous les sujets du quotidien académique, plus étroitement encore depuis son arrivée à la Sorbonne Nouvelle. La rigueur, la précision, le sérieux... sans esprit de sérieux, la simplicité, la justesse, l'élégance morale et intellectuelle caractérisaient sa manière d'être, d'agir, de penser et d'écrire. 